\title{
Microstructural Characterization of Molecular Assemblies by Digital- Imaging Cryo-TEM
}

\author{
Dganit Danino
}

Cell Biochemistry and Biology, NIDDK, NIH, Bethesda, MD

Macromolecular assemblies form in diverse systems including biological and biomedical solutions, polymers, surfactants and microemulsions. The assemblies are dynamic, and change their size and shape in response to changes in control parameters such as concentration, temperature, salinity and $\mathrm{pH}$. Detailed and aggregate-specific information about microstructured fluids can be obtained from direct imaging techniques. Cryo-TEM has been shown to be particularly useful in the study of molecular assemblies, as it is based on rapid thermal (physical) fixation that preserves the assemblies at their native state, and it provides clear and direct images of the structures at a resolution of $1 \mathrm{~nm}$.

We have recently developed digital-imaging cryo-TEM [1] that overcomes problems associated with imaging on photographic plates such as poor sampling, "shooting in the dark" to avoid radiation damage, and difficulties in accurate focusing as a result of heterogeneity of the vitrified film; difficulties that made cryo-TEM technically complicated and very inefficient, and limited its use significantly. In digital-imaging cryo-TEM, a side-mount CCD camera that operates at low dose conditions allows a preview of the exact area to be recorded, concentration on specific interesting features and adjustment of microscope parameters for optimal imaging. It also provides real-time feedback on specimen and image quality, and ease of post-microscopy image processing of the digitized images.

The CCD camera provides clear and direct images showing the size, shape and coexistence of different assemblies. It is especially advantageous for identifying infrequent assemblies, studying specific assembly details [2] and transient structures forming during dynamic processes [3]. This setup is also beneficial for studying highly radiation-sensitive systems such as oil-continuous solutions [4].

At present, most cryo electron microscopy studies are still performed using photographic film. We find it important to present our approach and procedures, with examples from particular studies that clearly demonstrate the advantages of the technique. The approach suggested here can be effectively and easily applied in many labs, as most modern microscopes are equipped with CCD cameras. We believe in the future cryo-TEM will eventually evolve into a solely digital image recording technique, and greatly broaden the number of systems examined. 
References

1. Danino D et al., Digital Cryogenic Transmission Electron Microscopy: an Advanced Tool for Direct Imaging of Complex Fluids, Colloids and Surfaces A: Physicochemical and Engineering Aspects 133, 113-122 (2001).

2. Simberg D et al., DNA Ordering in I.ipoplexes: Fffect of Different Tipid Composition, J Biol Chem. 276, 47453-47459 (2001).

3. Konikoff $\Gamma$ et al., Microstructural Evolution of Lipid Aggregates in Nucleating Model and Human Biles Visualized by Cryo-Transmission Electron Microscopy, Hepatology $31,261-268(2000)$.

4. Danino D et al., Direct Cryo-TEM Imaging of Phospholipid Aggregates in Soybean Oil ( JCIS, in press).
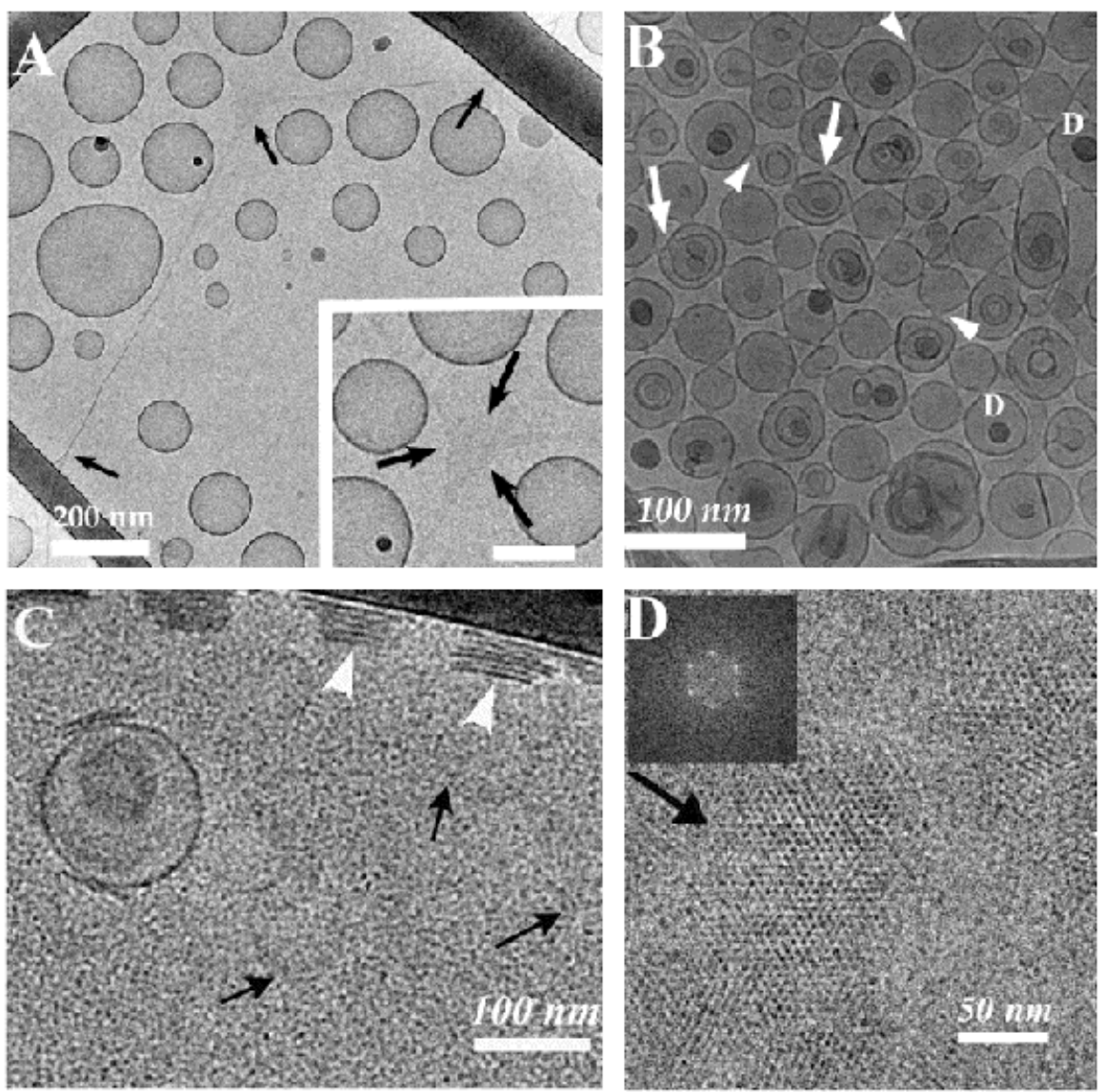

Figure 1: Examples of fine structural details obtained by digital-imaging cryo-TEM in a variety of complex fluid systems. (A) Three-fold locally flat junctions (arrows), connecting ribbon-like structures in a 3\% solution of the hybrid C8FC4-2-12 gemini surfactant. (B) Condensed DNA (D) and interconnected bilayer channels (arrowheads) in DNA/lipid complexes. (C) Transformation of spheroidal micelles through lamellar patches to vesicles, in nucleating bile. (D) A phospholipid (PL) liquid crystalline particle suspended in the oil phase of soybean oil/PL/ hexane mixture. The ГГT indicates a hexagonal ordering of this area. 